\title{
Using balances to engineer features for the classification of health biomarkers: a new approach to balance selection
}

\author{
Thomas P. Quinn ${ }^{1, *}$ and Ionas Erb² \\ ${ }^{1}$ Independent Scientist, Geelong, Australia \\ ${ }^{2}$ Centre for Genomic Regulation (CRG), The Barcelona Institute of Science and Technology, Barcelona, Spain \\ * contacttomquinn@gmail.com
}

\begin{abstract}
Since the turn of the century, technological advances have made it possible to obtain a molecular profile of any tissue in a cost-effective manner. Among these advances include sophisticated high-throughput assays that measure the relative abundance of microorganisms, RNA molecules, and metabolites. While these data are most often collected to gain new insights into biological systems, they can also be used as biomarkers to create clinically useful diagnostic classifiers. How best to classify high-dimensional "-omics" data remains an area of active research. However, few explicitly model the relative nature of these data, and instead rely on cumbersome normalizations which often invoke untestable assumptions. This report (a) emphasizes the relative nature of health biomarkers, (b) discusses the literature surrounding the classification of relative data, and (c) benchmarks how different transformations perform across multiple biomarker types. In doing so, this report explores how one could use balances to engineer features prior to classification, and proposes a simple procedure, called discriminative balance analysis, to select discriminative 2- and 3-part balances.
\end{abstract}

\section{Introduction}

Next-generation sequencing (NGS) technology is routinely used to quantify the presence of bacterial or gene species from environmental and biological samples. Hyphenated chromatographic assays like liquid chromatography-mass spectrometry (LC-MS) are used to quantify the presence of proteins, lipids, or metabolites. NGS and LC-MS both generate high-dimensional data that are used as health biomarkers to predict and surveil disease [28]. However, because NGS and LC-MS measure abundance by sampling from the total population, the total number of molecules recorded for a sample is arbitrary, thereby making these data compositional $[26,14,30,21,22,20,44]$. Others have already demonstrated that compositionality confounds the routine application of univariate [6], correlation [40], and distance [2] measures. Since machine learning pipelines often rely on these measures, compositionality may impact the accuracy of classifiers trained on these data [14, 24].

Compositional data analyses tend to have one of three flavours. First, the "simple" log-ratio approach uses a single reference to re-cast the data. Most commonly, the reference is the per-sample geometric mean (centered log-ratio $[\mathrm{clr}]$ transformation) or a single component (additive log-ratio [alr] transformation), but the geometric mean of inter-quartile range components [55] and of nonzero components [31] have also been proposed. After transformation, the analysis then proceeds as if the data were absolute, but with a caveat: the interpretation of the results depends on the reference used. Second, the "pragmatic approach" analyzes pairwise log-ratios directly, which has been used to score important genes [52] and gene pairs [23, 13], and to reduce the dimensionality of the data [23]. This approach makes sense when the ratios themselves have some importance to the analyst. However, it presents a clear problem for the classification of high-dimensional data: ratios "explode" feature space from $p=D$ features to $p=D(D-1) / 2$ (pairs of) features, compounding the $p>>N$ problem. Third, the "coordinate approach" uses an orthonormal basis to transform $D$ components into $D-1$ values via an isometric log-ratio (ilr) transformation [12]. When a serial binary partition (SBP) matrix is used to obtain the orthonormal basis, this approach yields a set 
of "balances", each of which describe a log-contrast between two sets of components [11, 49, 38]. Since the basis vectors used to make balances are associated with successive bipartitions of the original feature set, balances can be more interpretable than general log-contrasts, while still having the formal appeal of the ilr transformation (i.e., orthogonality of the basis vectors and a full-rank covariance matrix) $[11,7]$. However, the utility of balances depends on having a desirable SBP matrix, which must be manually curated or procedurally generated. One popular SBP, easily approximated via a simple heuristic, decomposes the variance such that the first balance explains the most variance, the second balance the second most, and so on [39, 32]. In microbiome research, authors have proposed using mean $\mathrm{pH}[35]$ and phylogenetics $[48,53]$ to construct an SBP.

Many publications have applied unsupervised statistical learning to compositional data. This includes the application of principal components analysis (PCA) [1], PCA biplots [4], and cluster analysis [33]. For PCA analysis, a clr-transformation is often used, although clr data do not have a full rank [15]. Instead, a robust PCA could be applied to ilr-transformed data, with the loadings back-transformed for interpretation [15].For cluster analysis, the Aitchison distance is preferred over the Euclidean distance, because only the former is sub-compositionally dominant [3, 33, 2]. Recently, Martin-Fernandez et al. have applied self-organized maps, a non-linear dimension reduction method, to ilr-transformed data [34], while Martino et al. have extended compositional PCA to zero-laden microbiome data [31]. Several studies have also applied supervised statistical learning to compositional data. Aitchison trained linear discriminant analysis (LDA) models on alr-transformed data [1], as have others [9] (though LDA is now usually applied to ilr-transformed data $[9,8])$. Generalized linear models, including logistic regression (LR), have also been used to classify composition data $[50,8]$. However, both LDA and LR require at least as many samples as features, making them inappropriate for high-dimensional health biomarker data (though this limitation is mitigated by regularization, used by [51] and [29] to classify compositions). Partial least squares (PLS), also suitable for high-dimensional data, has been applied to clr-transformed data to predict continuous outcomes [25], while PLS discriminant analysis (PLS-DA) has been used to classify clr-transformed [18] and ilr-transformed [27] data. In microbiome research, a step-wise algorithm, implemented as selbal, was proposed to identify a single balance that performs well in classification and regression tasks [46]. This latter work highlights an advantage to balances: although alr-, clr-, and ilr- transformations can facilitate statistical learning, balances can engineer the feature space into a single interpretable biomarker via balance selection.

How best to classify high-dimensional compositional data remains an open question. We are not aware of any work that benchmarks compositional data transformations as they pertain to the classification of high-dimensional compositional data. In this study, we employ a statistically robust cross-validation scheme to evaluate how well regularized LR classifies health-related binary outcomes on 13 compositional data sets. Specifically, we benchmark performance using features obtained from raw proportions, clr-transformed data, balances, and selected balances. Our results show that the centered log-ratio transformation, and all four balance procedures, outperform raw proportions for the classification of health biomarker data. We also propose a new balance selection procedure, called discriminatory balance analysis, that offers a computationally efficient way to select important 2- and 3-part balances. These discriminant balances reduce the feature space and improve the interpretability, without sacrificing classifier performance. In doing so, they also outperform a recently published balance selection method, selbal, in terms of run-time and classification accuracy.

\section{Methods}

\subsection{Data acquisition}

We acquired data from 4 principal sources. Two gut microbiome data sets (originally published in [19] and [37]) were acquired already quantified from the selbal package [46]. Two additional gut microbiome data sets (originally published in [47] and [5]) were acquired already quantified from the supplement of Duvallet et al. [10]. A fifth gut microbiome data set was acquired already quantified from the supplement of Franzosa et al. [16].

The Schubert et al. data contained 3-classes comparing hospital-acquired diarrhea (HAD) with community-acquired diarrhea $(\mathrm{CAD})$ and healthy controls. This data set was used in two tests: HAD vs. CAD and HAD vs. HC. The Buxter et al. data contained 3-classes comparing colorectal cancer (CRC) with adenoma (AC) and healthy controls (HC). This data set was also used in two 
tests: CRC vs. AC and CRC vs. HC. The Franzosa et al. data contained 3-classes comparing Crohn's disease (CD) and ulcerative colitis (UC) with healthy controls (HC). This data set was also used in two tests: (CD \& UC) vs. HC and CD vs. UC. Franzosa et al. also published gut metabolomic data for the same samples. These data were used for an additional two tests that parallel the gut microbiome tests.

A sixth data set was acquired from The Cancer Genome Atlas [54], and contains microRNA expression for primary breast cancer (BRCA) samples and healthy controls (HC). We further labelled the BRCA samples using PAM50 sub-types retrieved from the supplement of [36]. PAM50 uses a gene expression signature to assign an intrinsic sub-type to the primary breast cancer sample: sub-types include luminal A, luminal B, HER2-enriched, Basal, and Normal-like. These data were used in three tests: any BRCA vs. HC, HER2+ vs. all other BRCA, and LumA-BRCA vs. LumB-BRCA.

\subsection{Feature extraction and zero handling}

Before training any models, features with too few counts were removed from the data. For the metabolomic and microRNA data sets, only features within the top decile of total abundance were included (this was done to reduce the feature space so that selbal became computationally tractable). For all data sets, features that contained zeros in more than $90 \%$ of samples were excluded (this was done to remove biomarkers that are not reliably present in the data). Finally, zeros were replaced using a simple multiplicative replacement strategy via the zCompositions package (this was done because the Bayesian replacement strategy fails for heavily zero-laden data). Table 1 summarizes the tests used in this study.

\subsection{Data transformation}

Let us consider the data matrix $x_{i j}$ which describes the relative abundance of $j \in\{1, \ldots, D\}$ components (as features) across $i \in\{1, \ldots, N\}$ compositions (as samples). Since the data studied are compositional, they can be expressed as a sub-composition of parts of the whole. The closure operation expresses the data so that the measurements for each sample sum to 1 (i.e., as proportions). The closed data are benchmarked in this study as the point of reference:

$$
\operatorname{ACOMP}\left(\mathbf{x}_{i}\right)=\frac{\left[x_{i 1}, \ldots, x_{i D}\right]}{\sum_{j=1}^{D} x_{i j}}
$$

We also benchmark the popular centered log-ratio transformation (clr):

$$
\operatorname{CLR}\left(\mathbf{x}_{i}\right)=\log \left(\frac{\left[x_{i 1}, \ldots, x_{i D}\right]}{\sqrt[D]{\prod_{j=1}^{D} x_{i j}}}\right)
$$

We also use the isometric log-ratio transformation (ilr) to construct balances from a serial binary partition (SBP) matrix describing $z \in\{1, \ldots, D-1\} \log$-contrasts between $j \in\{1, \ldots, D\}$ parts. A log-contrast of a $D$-part composition is a log-linear combination $a_{1} \log x_{i 1}+\cdots+a_{D} \log x_{i D}$ with the constraint that $\sum_{j=1}^{D} a_{j}=0$. This constraint ensures scale invariance of the combination, i.e., normalization factors of $\mathbf{x}_{i}$ cancel.

Balances are constructed from special log-contrasts with $a_{j} \in\left\{\frac{1}{d^{+}}, \frac{-1}{d^{-}}, 0\right\}$ (where $d^{+}$and $d^{-}$ refer to the number of positive and negative entries in a column of the SBP matrix). Such logcontrasts have the form $\log \left(\left(\prod_{j \in \mathcal{C}^{+}} x_{i j}\right)^{1 / d^{+}} /\left(\prod_{k \in \mathcal{C}^{-}} x_{i k}\right)^{1 / d^{-}}\right)$where $\mathcal{C}^{+}$and $\mathcal{C}^{-}$are the sets of indices $j$ for which $a_{j}=\frac{1}{d^{+}}$and $a_{j}=\frac{-1}{d^{-}}$, respectively. The special form of these log-contrasts allows for a representation in form of a bi-partition. It is helpful to think of an SBP as a dendrogram tree, from which the terms $a_{j}$ can be derived (see Figure 1 for an example SBP). A balance value is now computed for each sample $i$ and each log-contrast $z$ :

$$
b_{i z}=\sqrt{\frac{d_{z}^{+} d_{z}^{-}}{d_{z}^{+}+d_{z}^{-}}} \log \left[\frac{\left(\prod_{j \in \mathcal{C}_{z}^{+}} x_{i j}\right)^{1 / d_{z}^{+}}}{\left(\prod_{k \in \mathcal{C}_{z}^{-}} x_{i k}\right)^{1 / d_{z}^{-}}}\right]
$$

The prefactor standardizes the log-contrasts such that $\sum_{j=1}^{D} a_{j z}^{2}=1$ and makes them orthogonal in the sense that $\sum_{j=1}^{D} a_{j z} a_{j z^{\prime}}=0$ for all $z \neq z^{\prime}$. 

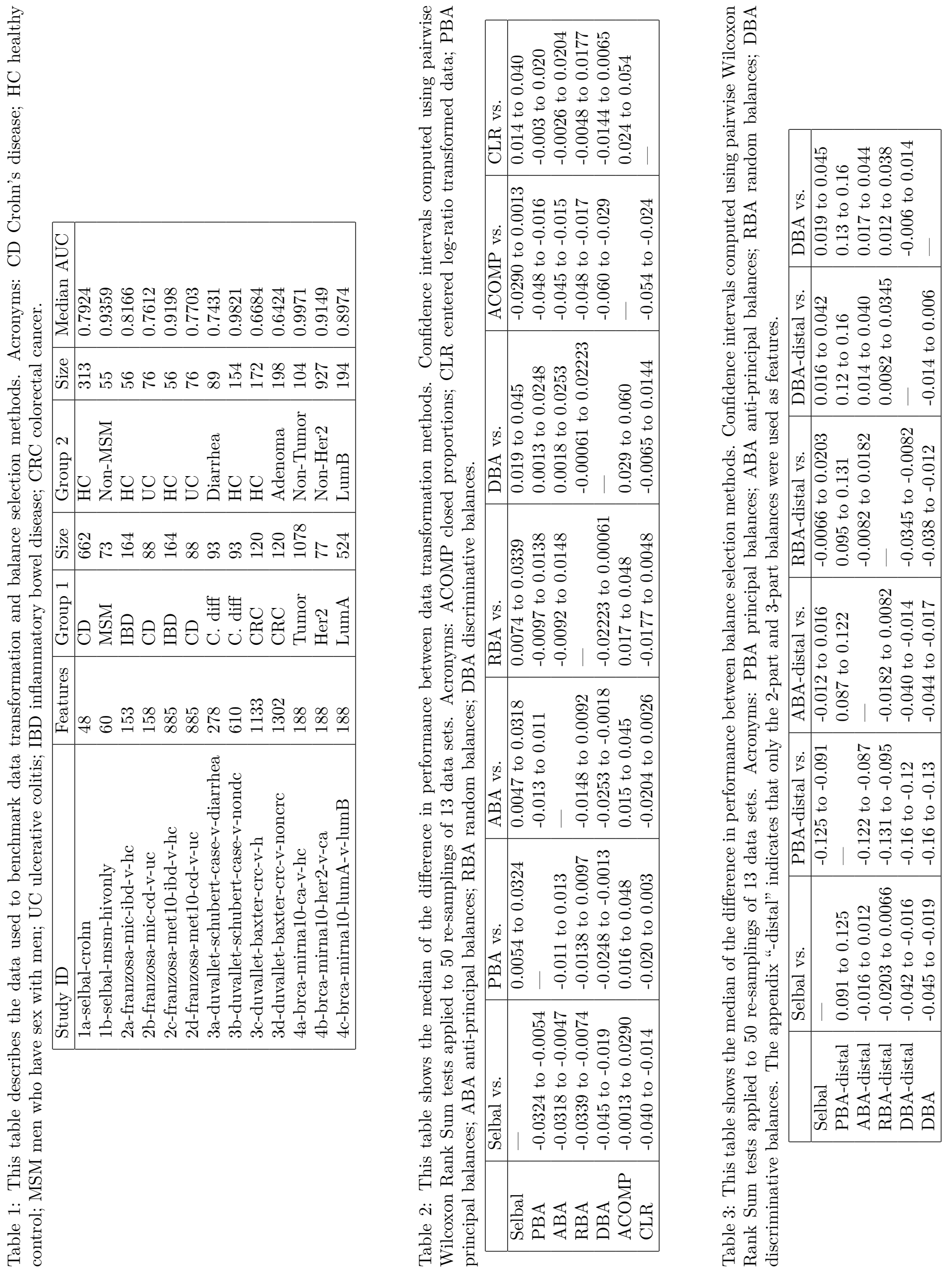


\subsection{The serial binary partition matrix}

We benchmark four procedures for generating an SBP. In PBA, we approximate a set of principal balances by hierarchically clustering the log-ratio variance matrix, $\mathbf{T}$, describing the relationship between any two variables $j$ and $j^{*}$ (see [39]):

$$
T_{j j^{*}}=\operatorname{var}\left[\log \frac{x_{1 j}}{x_{1 j^{*}}}, \ldots, \log \frac{x_{N j}}{x_{N j^{*}}}\right]
$$

Principal balances are analogous to principal components in that the first balance explains the most variance, the second balance the second most variance, and so on. Note that PBA only approximates principal balances.

In ABA, we hierarchically cluster a new dissimilarity measure defined as the difference of the $\log$-ratio variance matrix from the maximum log-ratio variance score: $\max (\mathbf{T})-T_{j j^{*}}$. In RBA, we generate random SBPs using a custom algorithm. In DBA, we generate an SBP that maximizes the discriminative potential of the distal branches. This is done by hierarchically clustering the differential proportionality matrix, $\Theta$, describing the relative contribution of the within-group logratio variances $\left(T_{j j^{*}}^{1}\right.$ and $\left.T_{j j^{*}}^{2}\right)$ to the total $\log$-ratio variance (see $[13,43]$ ):

$$
\theta_{j j^{*}}=\frac{N_{1} T_{j j^{*}}^{1}+N_{2} T_{j j^{*}}^{2}}{\left(N_{1}+N_{2}\right) T_{j j^{*}}}
$$

for groups sized $N_{1}$ and $N_{2}$. This matrix ranges from $[0,1]$ where 0 indicates that the two features have a maximally large difference in log-ratio means between the two group. Unlike the other SBP methods, the DBA method is supervised.

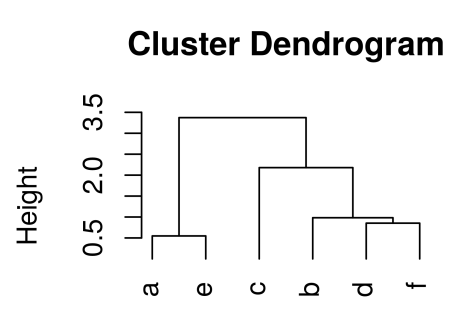

\begin{tabular}{|c|c|c|c|c|c|}
\hline $\mathbf{z 1}$ & $\mathbf{z 2}$ & $\mathbf{z 3}$ & $\mathbf{z 4}$ & $\mathbf{z 5}$ \\
\hline$a$ & 1 & 0 & 0 & 0 & 1 \\
\hline$b$ & -1 & -1 & 1 & 0 & 0 \\
\hline$c$ & -1 & 1 & 0 & 0 & 0 \\
\hline$d$ & -1 & -1 & -1 & 1 & 0 \\
\hline$e$ & 1 & 0 & 0 & 0 & -1 \\
\hline$f$ & -1 & -1 & -1 & -1 & 0 \\
\hline
\end{tabular}

\begin{tabular}{|c|c|c|c|}
\hline & b_and_d_and_f & d_and_f & a_and_e \\
\hline$a$ & 0 & 0 & 1 \\
\hline$b$ & 1 & 0 & 0 \\
\hline$c$ & 0 & 0 & 0 \\
\hline$d$ & -1 & 1 & 0 \\
\hline$e$ & 0 & 0 & -1 \\
\hline$f$ & -1 & -1 & 0 \\
\hline
\end{tabular}

Figure 1: This figure shows how a balance dendrogram relates to a serial binary partition (SBP) matrix. The left panel shows a dendrogram clustering the similarity between 6 components, where the first branch in the dendrogram refers to the first balance (i.e., [a \& e] vs. [c \& b \& d \& f]). The middle panel shows the corresponding SBP with 5 balances (columns) and the components involved in each log-contrast (rows). The right panel shows the "distal" 2- and 3-part balances.

Note that the SBP is always constructed using the training set only. The balance "rule" is then applied to the validation set prior to model deployment. All SBP procedures are implemented in the balance package with the functions sbp.fromPBA, sbp.fromABA, sbp.fromRandom, and sbp.fromPropd, respectively [42]. Differential proportionality analysis is implemented in the propr package [45] with the function propd.

\subsection{Classification pipeline}

In order to get a robust measure of performance, we repeat model training on 50 training sets randomly sampled from the data (with $33 \%$ set aside as a validation set). For each training set, we (1) transform features as described above, (2) train a model on the transformed features, (3) deploy the model on the withheld validation set, and (4) calculate the area under the receiver operating curve (AUC). AUC is used because it is more informative than accuracy when classes are imbalanced. Model splitting, transformation, training, and prediction are all handled by the 
high-throughput classification software exprso [41]. By repeating this procedure 50 times, we can calculate the median performance and its range.

When using selbal, a generalized linear model is trained on a single balance (as done in [46]). For all other transformations, a least absolute shrinkage and selection operator (LASSO) model is used to select features and fit the data simultaneously (via the glmnet package [17]). When using LASSO, $\lambda$ is chosen procedurally by measuring 5 -fold training set cross-validation accuracy over the series $\exp (\operatorname{seq}(\log (0.001), \log (5)$, length.out $=100))$, with the best $\lambda$ selected automatically by cv.glmnet.

\section{Results and Discussion}

\subsection{Choice in log-ratio transformation does not impact performance}

Figure 2 shows the validation set AUCs for binary classifiers trained on 13 data sets. In general, we see that the centered log-ratio transformation (CLR) and balance procedures (PBA, ABA, RBA, DBA) perform comparably. Although they all tend to outperform proportions (ACOMP), the proportions were more discriminative than the CLR for a few tests. This might occur when the closure bias itself confounds the predicted outcome.

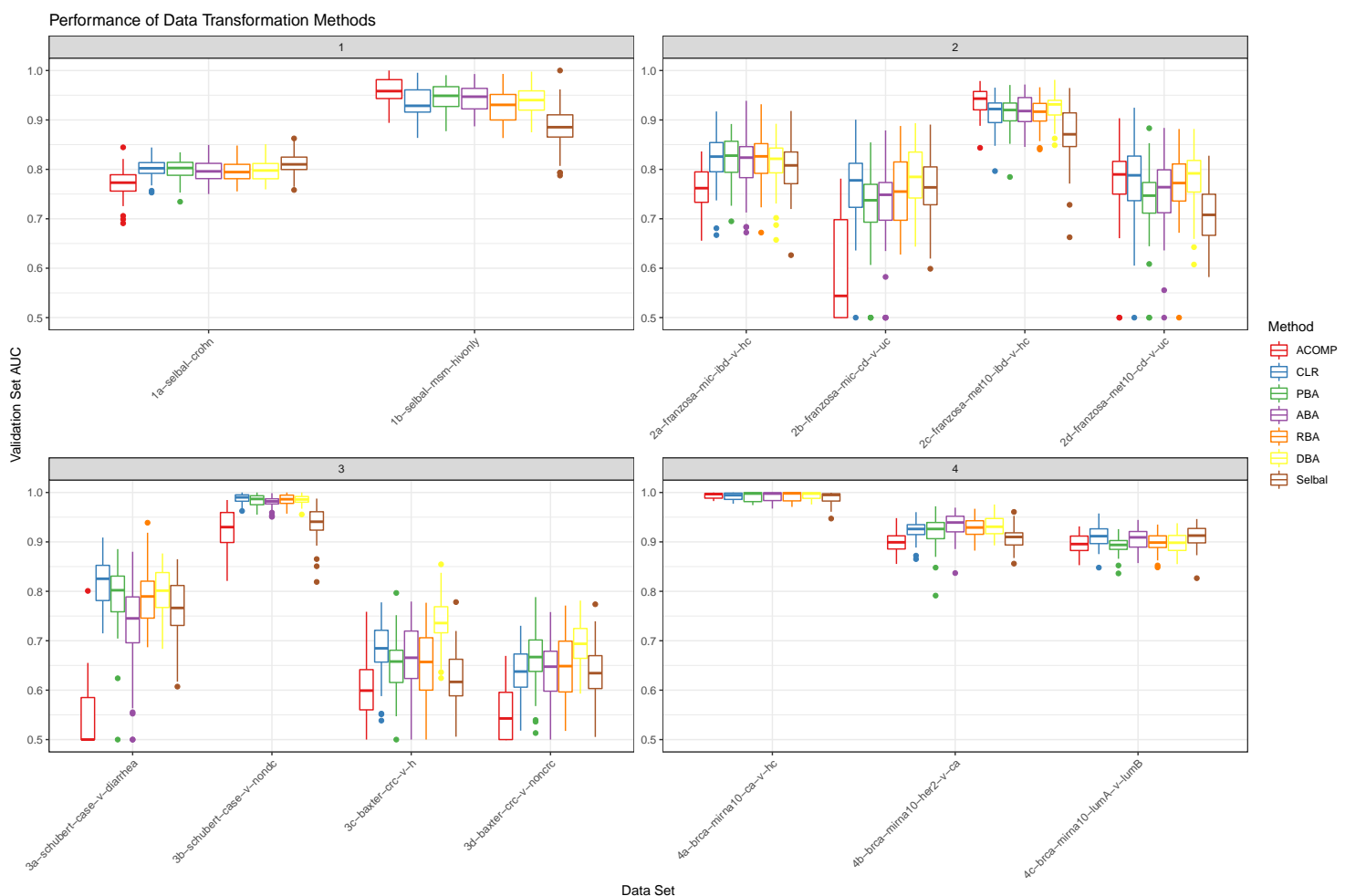

Figure 2: This figure shows the distribution of validation set AUCs (y-axis) for classifiers trained on closed or transformed data (x-axis). Each validation set AUC describes a unique random training and validation set split. All classifiers are regularized logistic regression models, with $\lambda$ tuned by training set cross-validation. Acronyms: ACOMP closed proportions; CLR centered log-ratio transformed data; PBA principal balances; ABA anti-principal balances; RBA random balances; DBA discriminative balances.

Table 2 shows the median of the difference between data transformations (as computed with pairwise Wilcoxon Rank Sum tests across all 13 tests). Here, we see that every transformation performs better than proportions. We also note that all balance procedures tend to perform equally well, though DBA balances perform marginally better. Although selbal posts an impressive accuracy for only using a single balance, it is less accurate than using a set of all balances. 


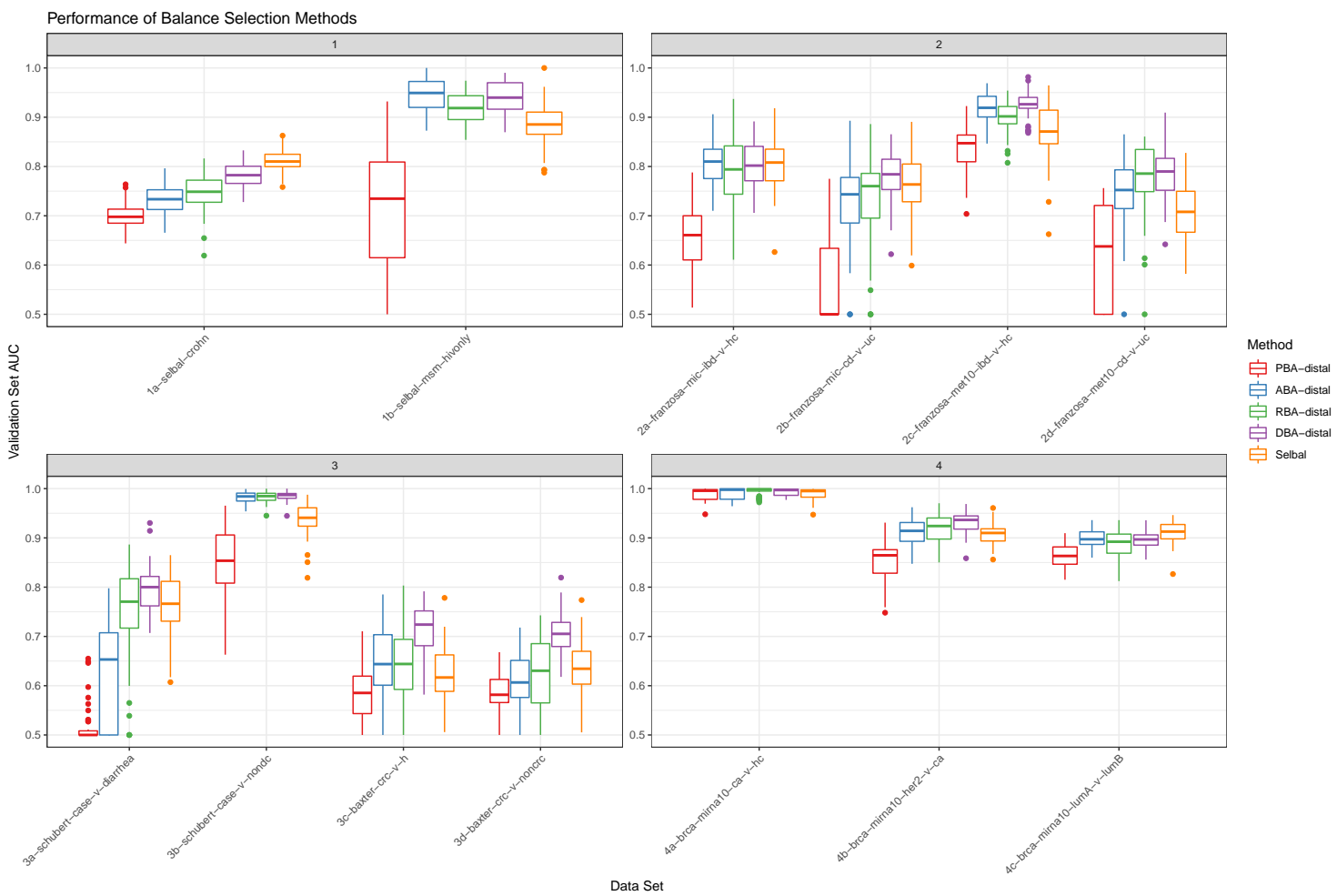

Figure 3: This figure shows the distribution of validation set AUCs (y-axis) for classifiers trained on selected balances (x-axis). Each validation set AUC describes a unique random training and validation set split. All classifiers are regularized logistic regression models, with $\lambda$ tuned by training set cross-validation. Acronyms: PBA principal balances; ABA anti-principal balances; RBA random balances; DBA discriminative balances. The appendix "-distal" indicates that only the 2-part and 3-part balances were used as features.

\subsection{DBA method selects predictive balances}

An advantage of using regularized logistic regression is that the model weights can be interpreted as a measure of feature importance. Even though the CLR and balances perform equally well, they imply different interpretations. While the CLR data do have a feature representing each component, the regularized weights do not describe the importance of that component. Rather, the CLR weights describe the importance of that variable relative to the sample mean. On the other hand, balances measure the log-contrast between sets of components. Thus, the balance weights describe the importance of those components directly.

For high-dimensional data, it can be challenging to interpret large balances. For example, the base of an SBP always contains one balance that is comprised of all variables. It may not be helpful in understanding the outcome to know that a log-contrast involving all components is discriminative. On the other hand, smaller balances (i.e., those involving fewer components) might have a clearer meaning to the analyst. Here, we propose a new procedure, called discriminative balance analysis (DBA), to generate an SBP that makes the smallest balances most discriminative. This procedure can be used to engineer and select important balances prior to model building. Since the selected balances contain few parts, they are more easily interpreted.

Conceptualizing the SBP as a tree, the largest balances are the "trunk" and the smallest balances are the "leaves" (see Figure 1). Since the basis is orthonormal, we can consider segments of the tree in the isolation of others. This also makes sure that the selected balances do not contain redundant information. Figure 3 shows classification AUC when only using the "distal leaf" balances (i.e., those with 2- or 3- parts). In principal balances analysis (PBA), the "trunk" contains the most variance, and the leaves the least. As expected, the distal PBA balances perform poorly. In anti-principal balance analysis (ABA), the "trunk" contains the least variance, and the leaves the most. As expected, the distal ABA balances outperform the distal PBA balances. In 
bioRxiv preprint doi: $\mathrm{https}$ ://doi.org/10.1101/600122; this version posted April 9, 2019. The copyright holder for this preprint (which was not certified by peer review) is the author/funder, who has granted bioRxiv a license to display the preprint in perpetuity. It is made available under aCC-BY-NC-ND 4.0 International license.
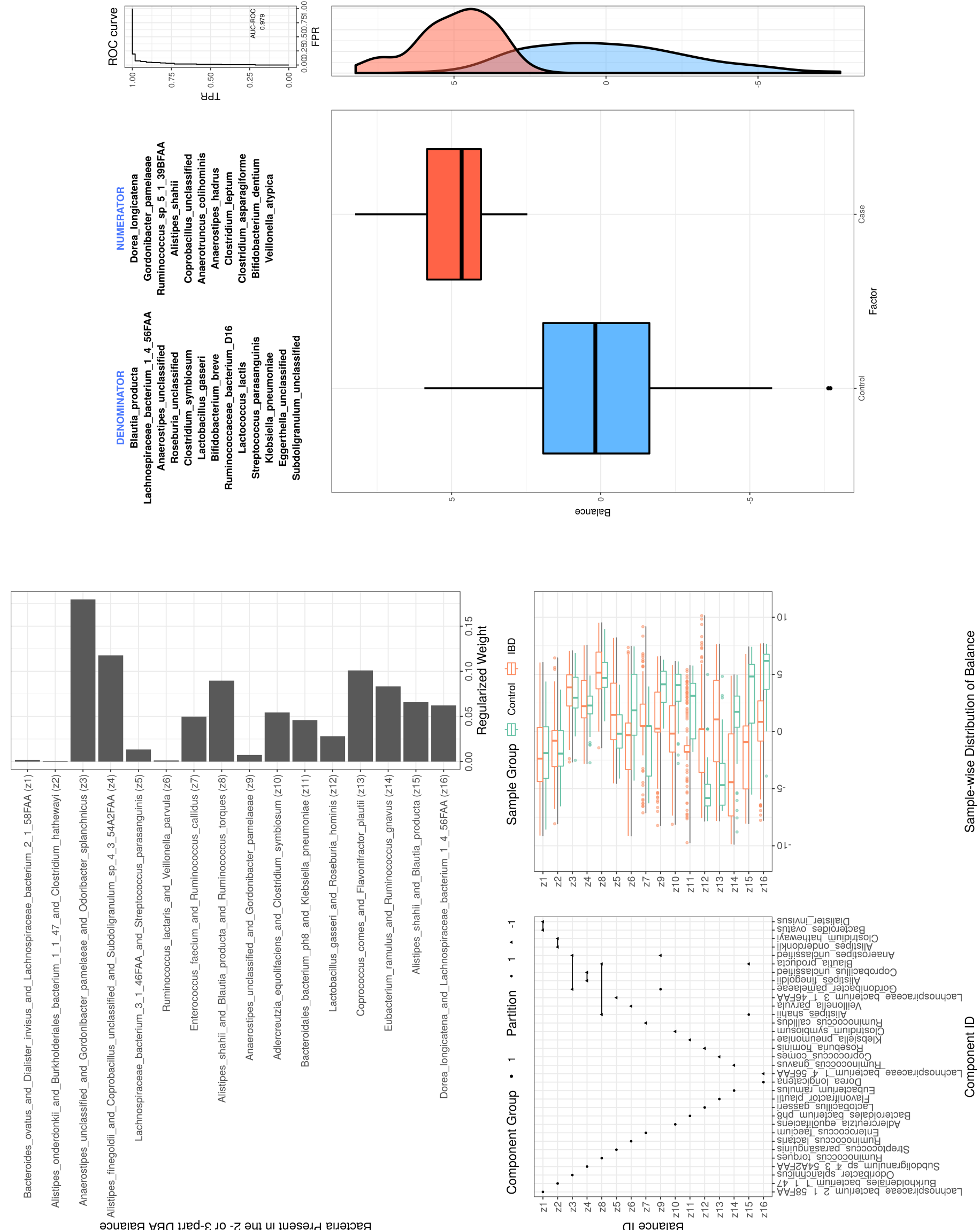

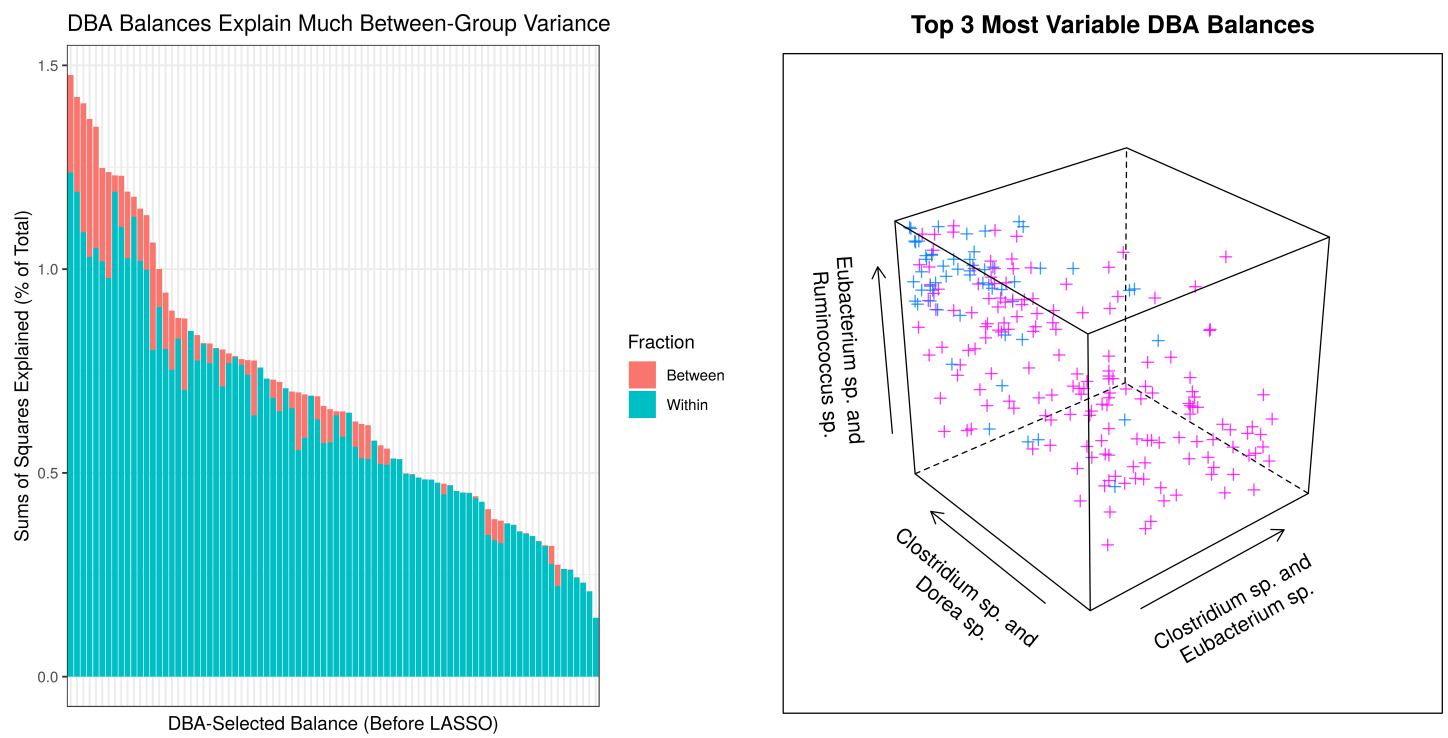

Figure 5: This figure shows the amount of variance (as a percent of the total) contained in each distal DBA balance (left panel), placed alongside a projection of the data across the top 3 most variable distal DBA balances (right panel). Good class separation is achieved using only 3 balances (each of which is proportional to a simple log-ratio). All panels generated using the 2a-franzosamic-ibd-v-hc data set.

discriminative balance analysis (DBA), the "trunk" is least discriminative, and the leaves the most. As expected, the distal DBA balances outperform both the PBA and ABA balances. Indeed, since DBA places the most discriminative balances distally, the distal DBA balances perform as well as all DBA balances (see Table 3 for $95 \%$ confidence interval). In random balance analysis (RBA), balances are random, so the leaves might be discriminative by chance. As expected, the distal RBA balances have an average performance.

The weights of DBA balances can be interpreted (and visualized) in an intuitive way. The 2-part balances can be visualized as a log-ratio, while the 3-part balances can be visualized with a ternary diagram or as a log-contrast. In Figure 4, we compare the most important distal DBA balances (left panel) with the single discriminative balance found by selbal (right panel). We see that many of the same variables are represented in both sets. However, DBA expresses the important variables via 2- and 3-part subsets that are, by definition of the SBP, grouped to be maximally discriminative. In the left panel, we see that balances with large regularized weights (top-left) have log-contrast scores that differentiate the groups (bottom-left). Though selbal performs remarkably well in its ability to select a single discriminative balance, our results suggest that the distal DBA method outperforms selbal by $\sim 1-4 \%$ AUC (see Table 3). Moreover, the distal DBA method is an order of magnitude faster than selbal, the latter of which must try multiple component combinations before finding the best log-contrast (25 minutes vs. 15 seconds for 1000 features).

\subsection{DBA as a discriminant ordination}

By using an orthonormal basis, balances decompose the variance into orthogonal parts. For a binary outcome, this allows us to not only calculate the amount of variance contained in each discriminative balance, but also to break down the contained variance into its between-group and within-group fractions (as done by an ANOVA). The left panel of Figure 5 shows that a large fraction of the (log-ratio) variance contained in the distal DBA balances is between-group variance. This is because the clustering of components using $\theta_{j j^{*}}$ as a distance will group together those components whose pairwise log-ratios describe only a small fraction of the within-group variance (i.e., a large fraction of between-group variance). Although we have already shown that the distal DBA balances are discriminative, the orthonormality of their underlying basis also allows us to use DBA to project a discriminant ordination of the data. In other words, we can visualize the data along mutually exclusive discriminant axes (similar to the axes in a discriminant analysis 
decomposing the variance between group means; however, for two groups, this would only give a single axis). The right panel of Figure 5 shows good class separation using only 3 balances (each of which is actually a simple log-ratio). From the left panel, we know that these three axes contain $4.3 \%$ of the total variance, and could likewise calculate that they contain $13.8 \%$ of the total between-group variance. Meanwhile, the DBA-selected balances together account for $90.4 \%$ of the total between-group variance. Yet, by restricting the ordination to the distal DBA balances, we ensure that each one of these discriminant axes is fully interpretable, having no more than 3 parts. On the other hand, if the analyst cared less about interpretation and more about maximizing contained between-group variance, they could do a clustering using as distance $1-\theta_{j j^{*}}$ and instead project the largest balances thus obtained (in direct analogy to the principal balances heuristic described above).

\section{Summary}

This work benchmarks the performance of regularized logistic regression classifiers across 13 highdimensional health biomarker data sets. Our results show that, on average, the centered log-ratio and balances both outperform raw proportions in classification tasks. We find that the serial binary partition (SBP) matrix used to generate the balances does not impact performance. However, the choice in SBP changes which balances are important for classification. In this report, we introduce a new SBP procedure that makes the most discriminative balances the smallest. This procedure, called discriminative balance analysis, offers a computationally efficient way to select important 2- and 3-part balances. These discriminant balances reduce the feature space and improve the interpretability, without sacrificing classifier performance. In doing so, they also outperform a recently published balance selection method, selbal, in terms of run-time and classification accuracy.

\section{Declarations}

\subsection{Ethics approval and consent to participate}

Not applicable.

\subsection{Consent for publication}

Not applicable.

\subsection{Availability of data and material}

All methods are available through open source software maintained by the authors.

\subsection{Competing interests}

No authors have competing interests.

\subsection{Funding}

Not applicable.

\subsection{Authors' contributions}

TPQ implemented the procedures, performed the analyses, and drafted the manuscript. IE derived the differential proportionality metric, contributed code, and expanded the manuscript. Both authors conceptualized the thesis and approved the final manuscript.

\section{Acknowledgements}

TPQ thanks the authors of selbal for inspiring this work. TPQ thanks Samuel C. Lee for his help with retrieving the TCGA data and the PAM50 labels. IE thanks Cedric Notredame for support. 


\section{References}

[1] J Aitchison. The Statistical Analysis of Compositional Data. Chapman \& Hall, Ltd., London, UK, UK, 1986.

[2] J. Aitchison, C. Barceló-Vidal, J. A. Martín-Fernández, and V. Pawlowsky-Glahn. Logratio Analysis and Compositional Distance. Mathematical Geology, 32(3):271-275, April 2000.

[3] John Aitchison. On criteria for measures of compositional difference. Mathematical Geology, 24(4):365-379, May 1992.

[4] John Aitchison and Michael Greenacre. Biplots of compositional data. Journal of the Royal Statistical Society: Series C (Applied Statistics), 51(4):375-392, October 2002.

[5] Nielson T. Baxter, Mack T. Ruffin, Mary A. M. Rogers, and Patrick D. Schloss. Microbiotabased model improves the sensitivity of fecal immunochemical test for detecting colonic lesions. Genome Medicine, 8(1):37, April 2016.

[6] K. Gerald van den Boogaart and Raimon Tolosana-Delgado. Descriptive Analysis of Compositional Data. In Analyzing Compositional Data with $R$, Use R!, pages 73-93. Springer, Berlin, Heidelberg, 2013.

[7] K. Gerald van den Boogaart and Raimon Tolosana-Delgado. Fundamental Concepts of Compositional Data Analysis. In Analyzing Compositional Data with R, Use R!, pages 13-50. Springer Berlin Heidelberg, 2013.

[8] K. Gerald van den Boogaart and Raimon Tolosana-Delgado. Multivariate Statistics. In Analyzing Compositional Data with R, Use R!, pages 177-207. Springer, Berlin, Heidelberg, 2013.

[9] Gareth P. Campbell, James M. Curran, Gordon M. Miskelly, Sally Coulson, Gregory M. Yaxley, Eric C. Grunsky, and Simon C. Cox. Compositional data analysis for elemental data in forensic science. Forensic Science International, 188(1-3):81-90, July 2009.

[10] Claire Duvallet, Sean M. Gibbons, Thomas Gurry, Rafael A. Irizarry, and Eric J. Alm. Metaanalysis of gut microbiome studies identifies disease-specific and shared responses. Nature Communications, 8(1):1784, December 2017.

[11] J. J. Egozcue and V. Pawlowsky-Glahn. Groups of Parts and Their Balances in Compositional Data Analysis. Mathematical Geology, 37(7):795-828, October 2005.

[12] J. J. Egozcue, V. Pawlowsky-Glahn, G. Mateu-Figueras, and C. Barceló-Vidal. Isometric Logratio Transformations for Compositional Data Analysis. Mathematical Geology, 35(3):279300, April 2003.

[13] Ionas Erb, Thomas Quinn, David Lovell, and Cedric Notredame. Differential Proportionality - A Normalization-Free Approach To Differential Gene Expression. Proceedings of CoDaWork 2017, The 7th Compositional Data Analysis Workshop; available under bioRxiv, page 134536, May 2017.

[14] P. Filzmoser and B. Walczak. What can go wrong at the data normalization step for identification of biomarkers? Journal of Chromatography. A, 1362:194-205, October 2014.

[15] Peter Filzmoser, Karel Hron, and Clemens Reimann. Principal component analysis for compositional data with outliers. Environmetrics, 20(6):621-632, September 2009.

[16] Eric A. Franzosa, Alexandra Sirota-Madi, Julian Avila-Pacheco, Nadine Fornelos, Henry J. Haiser, Stefan Reinker, Tommi Vatanen, A. Brantley Hall, Himel Mallick, Lauren J. McIver, Jenny S. Sauk, Robin G. Wilson, Betsy W. Stevens, Justin M. Scott, Kerry Pierce, Amy A. Deik, Kevin Bullock, Floris Imhann, Jeffrey A. Porter, Alexandra Zhernakova, Jingyuan Fu, Rinse K. Weersma, Cisca Wijmenga, Clary B. Clish, Hera Vlamakis, Curtis Huttenhower, and Ramnik J. Xavier. Gut microbiome structure and metabolic activity in inflammatory bowel disease. Nature Microbiology, December 2018. 
[17] Jerome Friedman, Trevor Hastie, and Rob Tibshirani. Regularization Paths for Generalized Linear Models via Coordinate Descent. Journal of Statistical Software, 33(1):1-22, 2010.

[18] Michele Gallo. Discriminant partial least squares analysis on compositional data. Statistical Modelling, 10(1):41-56, April 2010.

[19] Dirk Gevers, Subra Kugathasan, Lee A. Denson, Yoshiki Vázquez-Baeza, Will Van Treuren, Boyu Ren, Emma Schwager, Dan Knights, Se Jin Song, Moran Yassour, Xochitl C. Morgan, Aleksandar D. Kostic, Chengwei Luo, Antonio González, Daniel McDonald, Yael Haberman, Thomas Walters, Susan Baker, Joel Rosh, Michael Stephens, Melvin Heyman, James Markowitz, Robert Baldassano, Anne Griffiths, Francisco Sylvester, David Mack, Sandra Kim, Wallace Crandall, Jeffrey Hyams, Curtis Huttenhower, Rob Knight, and Ramnik J. Xavier. The treatment-naive microbiome in new-onset Crohn's disease. Cell Host 8 Microbe, 15(3):382-392, March 2014.

[20] Gregory B. Gloor, Jean M. Macklaim, Vera Pawlowsky-Glahn, and Juan J. Egozcue. Microbiome Datasets Are Compositional: And This Is Not Optional. Frontiers in Microbiology, 8, 2017.

[21] Gregory B. Gloor, Jia Rong Wu, Vera Pawlowsky-Glahn, and Juan José Egozcue. It's all relative: analyzing microbiome data as compositions. Annals of Epidemiology, 26(5):322-329, May 2016.

[22] Gregory Brian Gloor, Jean M. Macklaim, Michael Vu, and Andrew D. Fernandes. Compositional uncertainty should not be ignored in high-throughput sequencing data analysis. Austrian Journal of Statistics, 45(4):73-87, July 2016.

[23] Michael Greenacre. Variable Selection in Compositional Data Analysis Using Pairwise Logratios. Mathematical Geosciences, pages 1-34, July 2018.

[24] Henry Han and Ke Men. How does normalization impact RNA-seq disease diagnosis? Journal of Biomedical Informatics, 85:80-92, September 2018.

[25] John Hinkle and William Rayens. Partial least squares and compositional data: problems and alternatives. Chemometrics and Intelligent Laboratory Systems, 30(1):159-172, November 1995.

[26] Hana Janečková, Karel Hron, Petr Wojtowicz, Eva Hlídková, Anna Barešová, David Friedecký, Lenka Zídková, Petr Hornik, Darina Behúlová, Dagmar Procházková, Hana Vinohradská, Karolína Pešková, Per Bruheim, Vratislav Smolka, Sylvie Stastná, and Tomáš Adam. Targeted metabolomic analysis of plasma samples for the diagnosis of inherited metabolic disorders. Journal of Chromatography. A, 1226:11-17, February 2012.

[27] Alžběta Kalivodová, Karel Hron, Peter Filzmoser, Lukáš Najdekr, Hana Janečková, and Tomáš Adam. PLS-DA for compositional data with application to metabolomics. Journal of Chemometrics, 29(1):21-28, January 2015.

[28] Pedro Larrañaga, Borja Calvo, Roberto Santana, Concha Bielza, Josu Galdiano, Iñaki Inza, José A. Lozano, Rubén Armañanzas, Guzmán Santafé, Aritz Pérez, and Victor Robles. Machine learning in bioinformatics. Briefings in Bioinformatics, 7(1):86-112, March 2006.

[29] Wei Lin, Pixu Shi, Rui Feng, and Hongzhe Li. Variable selection in regression with compositional covariates. Biometrika, 101(4):785-797, December 2014.

[30] David Lovell, Vera Pawlowsky-Glahn, Juan José Egozcue, Samuel Marguerat, and Jürg Bähler. Proportionality: A Valid Alternative to Correlation for Relative Data. PLoS Computational Biology, 11(3), March 2015.

[31] Cameron Martino, James T. Morton, Clarisse A. Marotz, Luke R. Thompson, Anupriya Tripathi, Rob Knight, and Karsten Zengler. A Novel Sparse Compositional Technique Reveals Microbial Perturbations. mSystems, 4(1):e00016-19, February 2019.

[32] J. A. Martín-Fernández, V. Pawlowsky-Glahn, J. J. Egozcue, and R. Tolosona-Delgado. Advances in Principal Balances for Compositional Data. Mathematical Geosciences, 50(3):273298, April 2018. 
[33] JA Martín-Fernández, C Barceló-Vidal, V Pawlowsky-Glahn, A Buccianti, G Nardi, and R Potenza. Measures of difference for compositional data and hierarchical clustering methods. In Proceedings of IAMG, volume 98, pages 526-531, 1998.

[34] Josep A. Martín-Fernández, Mark A. Engle, Leslie F. Ruppert, and Ricardo A. Olea. Advances in self-organizing maps for their application to compositional data. Stochastic Environmental Research and Risk Assessment, February 2019.

[35] James T. Morton, Jon Sanders, Robert A. Quinn, Daniel McDonald, Antonio Gonzalez, Yoshiki Vázquez-Baeza, Jose A. Navas-Molina, Se Jin Song, Jessica L. Metcalf, Embriette R. Hyde, Manuel Lladser, Pieter C. Dorrestein, and Rob Knight. Balance Trees Reveal Microbial Niche Differentiation. mSystems, 2(1):e00162-16, February 2017.

[36] Dvir Netanely, Ayelet Avraham, Adit Ben-Baruch, Ella Evron, and Ron Shamir. Expression and methylation patterns partition luminal-A breast tumors into distinct prognostic subgroups. Breast Cancer Research, 18(1):74, July 2016.

[37] Marc Noguera-Julian, Muntsa Rocafort, Yolanda Guillén, Javier Rivera, Maria Casadellà, Piotr Nowak, Falk Hildebrand, Georg Zeller, Mariona Parera, Rocío Bellido, Cristina Rodríguez, Jorge Carrillo, Beatriz Mothe, Josep Coll, Isabel Bravo, Carla Estany, Cristina Herrero, Jorge Saz, Guillem Sirera, Ariadna Torrela, Jordi Navarro, Manel Crespo, Christian Brander, Eugènia Negredo, Julià Blanco, Francisco Guarner, Maria Luz Calle, Peer Bork, Anders Sönnerborg, Bonaventura Clotet, and Roger Paredes. Gut Microbiota Linked to Sexual Preference and HIV Infection. EBioMedicine, 5:135-146, March 2016.

[38] Vera Pawlowsky-Glahn and Juan Jose Egozcue. Exploring Compositional Data with the CoDa-Dendrogram. Austrian Journal of Statistics, 40(1\&2):103-113, 2011.

[39] Vera Pawlowsky-Glahn, Juan José Egozcue, and Raimon Tolosana Delgado. Principal balances. Proceedings of CoDaWork 2011, The 4th Compositional Data Analysis Workshop, pages $1-10,2011$.

[40] Karl Pearson. Mathematical Contributions to the Theory of Evolution. III. Regression, Heredity, and Panmixia. Philosophical Transactions of the Royal Society of London. Series A, Containing Papers of a Mathematical or Physical Character, 187:253-318, 1896.

[41] Thomas Quinn, Daniel Tylee, and Stephen Glatt. exprso: an R-package for the rapid implementation of machine learning algorithms. F1000Research, 5:2588, December 2017.

[42] Thomas P. Quinn. Visualizing balances of compositional data: A new alternative to balance dendrograms. F1000Research, 7:1278, August 2018.

[43] Thomas P. Quinn, Ionas Erb, Greg Gloor, Cedric Notredame, Mark F. Richardson, and Tamsyn M. Crowley. A field guide for the compositional analysis of any-omics data. bioRxiv, page 484766, December 2018.

[44] Thomas P. Quinn, Ionas Erb, Mark F. Richardson, Tamsyn M. Crowley, and Jonathan Wren. Understanding sequencing data as compositions: an outlook and review. Bioinformatics, 34(16):2870-2878, August 2018.

[45] Thomas P. Quinn, Mark F. Richardson, David Lovell, and Tamsyn M. Crowley. propr: An R-package for Identifying Proportionally Abundant Features Using Compositional Data Analysis. Scientific Reports, 7(1):16252, November 2017.

[46] J. Rivera-Pinto, J. J. Egozcue, V. Pawlowsky-Glahn, R. Paredes, M. Noguera-Julian, and M. L. Calle. Balances: a New Perspective for Microbiome Analysis. mSystems, 3(4):e0005318, August 2018.

[47] Alyxandria M. Schubert, Mary A. M. Rogers, Cathrin Ring, Jill Mogle, Joseph P. Petrosino, Vincent B. Young, David M. Aronoff, and Patrick D. Schloss. Microbiome Data Distinguish Patients with Clostridium difficile Infection and Non-C. difficile-Associated Diarrhea from Healthy Controls. mBio, 5(3):e01021-14, July 2014. 
[48] Justin D. Silverman, Alex D. Washburne, Sayan Mukherjee, and Lawrence A. David. A phylogenetic transform enhances analysis of compositional microbiota data. eLife, 6, 2017.

[49] S. Thió-Henestrosa, J. J. Egozcue, V. Pawlowsky-Glahn, L. Ó. Kovács, and G. P. Kovács. Balance-dendrogram. A new routine of CoDaPack. Computers $\&$ Geosciences, 34(12):16821696, December 2008.

[50] Raimon Tolosana Delgado. Uses and misuses of compositional data in sedimentology. Sedimentary geology, 280(S.I):60-79, December 2012.

[51] Michail T. Tsagris, Simon Preston, and Andrew T. A. Wood. A data-based power transformation for compositional data. arXiv:1106.1451 [stat], June 2011. arXiv: 1106.1451.

[52] Jan Walach, Peter Filzmoser, Karel Hron, Beata Walczak, and Lukáš Najdekr. Robust biomarker identification in a two-class problem based on pairwise log-ratios. Chemometrics and Intelligent Laboratory Systems, 171:277-285, December 2017.

[53] Alex D. Washburne, Justin D. Silverman, Jonathan W. Leff, Dominic J. Bennett, John L. Darcy, Sayan Mukherjee, Noah Fierer, and Lawrence A. David. Phylogenetic factorization of compositional data yields lineage-level associations in microbiome datasets. PeerJ, 5:e2969, 2017.

[54] John N. Weinstein, Eric A. Collisson, Gordon B. Mills, Kenna M. Shaw, Brad A. Ozenberger, Kyle Ellrott, Ilya Shmulevich, Chris Sander, and Joshua M. Stuart. The Cancer Genome Atlas Pan-Cancer Analysis Project. Nature genetics, 45(10):1113-1120, October 2013.

[55] Jia R. Wu, Jean M. Macklaim, Briana L. Genge, and Gregory B. Gloor. Finding the centre: corrections for asymmetry in high-throughput sequencing datasets. arXiv:1704.01841 [q-bio], April 2017. arXiv: 1704.01841. 